OPEN ACCESS

Edited by:

Karina Vold

University of Toronto, Canada

Reviewed by: Vinaya Manchaiah,

Lamar University, United States Giovanna Nunes Vilaza,

Technical University of

Denmark, Denmark

*Correspondence:

Camille Nebeker

Nebeker@eng.ucsd.edu

Specialty section

This article was submitted to Human Factors and Digital Health,

a section of the journal

Frontiers in Digital Health

Received: 04 April 2021 Accepted: 17 June 2021 Published: 15 July 2021

Citation:

Nebeker C, Gholami M, Kareem D and Kim E (2021) Applying a Digital Health Checklist and Readability Tools

to Improve Informed Consent for

Digital Health Research.

Front. Digit. Health 3:690901.

doi: 10.3389/fdgth.2021.690901

\section{Applying a Digital Health Checklist and Readability Tools to Improve Informed Consent for Digital Health Research}

\author{
Camille Nebeker ${ }^{1,2 *}$, Maryam Gholami ${ }^{1,2}$, Daniah Kareem ${ }^{1,2}$ and Emily Kim ${ }^{1}$ \\ ${ }^{1}$ Research Center for Optimal Digital Ethics in Health, Herbert Wertheim School of Public Health and Human Longevity \\ Science, University of California, San Diego, La Jolla, CA, United States, ${ }^{2}$ Design Lab, University of California, San Diego, \\ La Jolla, CA, United States
}

Background: As research involving human participants increasingly occurs with the aid of digital tools (e.g., mobile apps, wearable and remote pervasive sensors), the consent content and delivery process is changing. Informed consent documents to participate in research are lengthy and difficult for prospective participants to read and understand. As the consent communication will need to include concepts and procedures unique to digital health research, making that information accessible and meaningful to the prospective participant is critical for consent to be informed. This paper describes a methodology that researchers can apply when developing a consent communication for digital health research.

Methods: A consent document approved by a US institutional review board was deconstructed into segments that aligned with federal requirements for informed consent. Three researchers independently revised each segment of text with a goal of achieving a readability score between a 6-8th grade level. The team then consulted with an external readability expert well-versed in revising informed consent documents into "plain language." The resulting text was evaluated using Microsoft Word and OnlineUtility accessibility software. The final step involved adding visual images and graphics to complement the text. The Digital Health Checklist consent prototype builder was then used to identify areas where the consent content could be expanded to address four key domains of Access and Usability, Privacy, Risks and Benefits, and Data Management.

Results: The approved consent was evaluated at a 12.6 grade reading level, whereas the revised language by our study team received $12.4,12$, and 12.58 , respectively. The final consent document synthesized the most readable of the three revised versions and was further revised to include language recommended by the software tool for improving readability, which resulted in a final revised consent readability score of a 9.2 grade level. Moreover, word count was reduced from 6,424 in the original consent to 679 in the rewritten consent form.

Conclusion: Utilizing an iterative process to design an accessible informed consent document is a first step in achieving meaningful consent to participate in digital health research. This paper describes how a consent form approved by an institutional review 
board can be made more accessible to a prospective research participant by improving the document readability score, reducing the word count and assessing alignment with the Digital Health Checklist.

Keywords: informed consent, digital health, internal review board, human factors, human centered design, meaningful consent, Digital Health Checklist tool

\section{INTRODUCTION}

In biomedical and behavioral research conducted by regulated entities, obtaining the prospective informed consent of those who become participants in research a cornerstone of ethical research. The purpose of informed consent is to provide people who are considering whether to participate in research the information necessary to determine if they want to volunteer (1). The regulations along with principles described in the Belmont Report intended to guide ethical research are used to determine what information is typically presented in the consent document $(1,2)$. The US Federal Regulation for Human Research Protections (see 45 CFR 46.116) lists eight key areas that must be described within the consent form (i.e., purpose, experimental aspects, risks, benefits, etc.). In addition to content requirements, there are guidelines suggesting that consent language be accessible aiming for a 6-8th grade reading level and presented in a setting whereby the individual is able to consider the information without undue influence that may compromise their ability to volunteer.

While informed consent is a demonstration of the ethical principle of "Respect for Persons," in reality, the practice of composing, delivering and obtaining consent to participate in research is far from perfect. Some of the problems stem from assumptions we, as researchers, make as we engage in what is typically a transactional conversation with a prospective research participant. This dialogue begins with the researcher stating they are conducting research to answer an important question followed by details about who is eligible, what's involved, how data will be collected and so forth. The first problem, which is not trivial, is the assumption that people understand what the scientific method involves; however, many don't and, subsequently misunderstandings follow (3). For example, researchers found that even when people can explain what a study involves, they may experience therapeutic misconception, and believe they will receive a medical care (4). In addition to barriers due to consent content, how it's delivered can also presented challenges for achieving informed consent. A number of studies have looked at steps to improving the consent process for example, among older adults (5), with cognitively impaired individuals (6) and adolescents (7). Yet, as we venture into the digital age, more is needed before we can be confident that informed consent is truly informed (8).

As research involving human participants increasingly occurs with the aid of digital tools (e.g., mobile apps, wearable and remote pervasive sensors), the consent content and delivery process is changing. Using digital strategies, researchers can now recruit and enroll upwards of 20,000+ participants rapidly to study various health conditions. The mPower study is one example where Apple Research Kit was used to host a mobile health study focused on Parkinson's disease with a goal of enrolling 20,000 participants using a mobile e-consent process (9). There was no one-on-one conversation between the researcher and prospective participants-all consent information was delivered remotely by placing information and graphics on the prospective participant's smartphone screen. Wearable and home placed sensors are another method used to passively gather a participant's personal health data in their natural environment, unobtrusively. The challenge with studies that take place using social media platforms, mobile apps or other forms of passive, remote study engagement is in how the researcher conveys the complex concepts of digital data collection or technology delivered interventions so that participants actually understand what participation involves. In the digital research environment, not only is explaining the concept of research important but, addressing potential technology and data literacy challenges is also important $(10,11)$. While the literature reflects ongoing persistent challenges with the concept of informed consent, little guidance is available to support those in the digital health research community who are working to fit a square peg (current consent paradigm) into a round hole (emerging digital health modalities).

The intent of this paper is to provide guidance to digital health researchers on how they can improve the informed consent communication specific to digital health research. This paper describes the process of developing an accessible consent communication. To demonstrate the process, we used the IRBapprove informed consent document developed for a study that involves body worn sensors to capture natural behaviors between a mom and baby in the home environment. In addition, a new checklist tool and framework to guide the consent deconstruction and reconstruction process was used. The Digital Health Checklist (DHC) is a decision support tool developed with a goal of supporting digital health researchers to design safe and responsible digital health research studies (12), including the content developed for use in obtaining informed consent.

\section{METHODS \\ The DHC Tool}

The DHC was developed via an iterative participatory design process to support decision making during the research protocol and consent development process prior to submission to an ethics review committee [e.g., Institutional Review Board (IRB in the US) or Research Ethics Committee (REC in Canada, European Union)] (12). Given the new challenges in developing ethical digital health studies (e.g., privacy considerations, data management and consent) (see Figure 1), the DHC tool prompts 


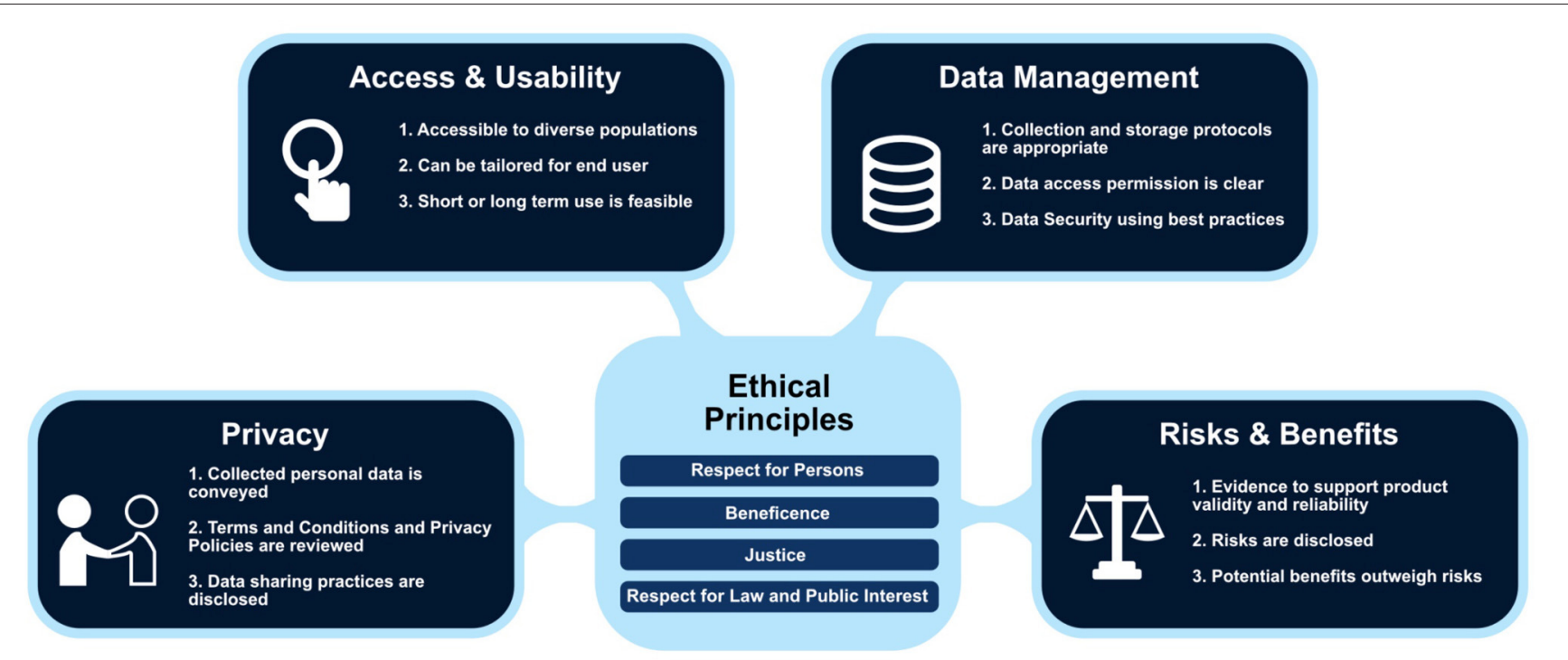

FIGURE 1 | Digital Health Framework with examples of checklist prompts embedded within each domain (Used with permission of C. Nebeker, ReCODE Health).

the researcher to consider factors that can influence responsible and safe research practices. The DHC is undergirded by a framework grounded in accepted ethical principles of respect for persons, beneficence and justice (1) augmented by a fourth principle of respect for law and public interest (13). The checklist items are depicted in a matrix table with the vertical listing ethical principles with the horizontal listed the four domains of: Access and Usability, Privacy, Risks and Benefits, and Data Management. For this prototype design process, we used the "respect for persons" section of the DHC as a blueprint to guide the consent content.

\section{Study Design}

This consent design process was initiated to support a longitudinal observational research study that would involve women and children as participants. Specifically, the US National Institutes of Health (NIH) funded a network of researchers to plan the HEALthy Brain and Child Development (HBCD) Study ${ }^{1}$. HBCD will examine early neurological development after prenatal exposure to maternal substance use using a variety of measures, including wearable and remote sensor technologies. The first author was part of an ethics and law working group involved with identifying and examining the ethical, legal and social implications associated with this potentially controversial research. Two passive sensor technologies were being considered for use during the study planning phase. One was a remote sensor that would be placed on the legs of a baby crib/bed to gather respiration and heart rate (14). The other, a body placed sensor that would be worn by the mother and baby to passively collect interpersonal data for specified periods in the home environment. Of the two devices, we selected the IRB-approved consent document developed for the

${ }^{1}$ https://heal.nih.gov/research/infants-and-children/healthy-brain wearable sensor since it involved prospective data collection from research participants.

This study involved a 4-step iterative human-centered design (HCD) process (15). The 4-step HCD include: (1) Understand and Address the Core Problems, to solve the fundamental issues, not the symptoms. (2) Be People-Centered, as opposed to technology-centered, ensuring that the outcome is appropriate for the culture and environment. (3) Use an ActivityCentered Systems Approach, focus upon the entire activity under consideration. (4) Rapid Iterations of Prototyping and Testing, and then refine and enhance the capabilities through successive iterations. While application of the HCD process is novel for developing a more accessible consent, we found it useful in conveying our process of developing an accessible consent communication.

\section{Understand and Address the Core Problems}

The content of informed consent communications used in regulated research is dictated by the federal regulations specific to human subject protections and local Institutional Review Boards. These documents include basic information that an individual may use to decide whether to participate in a research study. Consent communications have become increasingly transactional and include legal disclaimers on top of the basic information about research study participation (e.g., purpose, procedures, risks, benefits, data management, conflicts of interest). This has added to the length of consent communications and has elevated the reading level to around a 10-12th grade, making it difficult for many readers to comprehend $(16,17)$. To understand a consent communication for digital health research, there are added complexities in that the reader will need, in addition to a level of research literacy, a level of technology and data literacy (18). 
TABLE 1 | US federal regulations state that the following information be conveyed to prospective research participants prior to enrolling as a volunteer in research.

\begin{tabular}{|c|c|}
\hline Statement that describes: & Detail needed \\
\hline - Research study involvement & $\begin{array}{l}\text { Explain the purpose, expected duration of participation, what procedures will be followed and a description of } \\
\text { experimental aspects. }\end{array}$ \\
\hline - Study benefits & Describe any direct benefits to the participant or others, which may be anticipated. \\
\hline - Study risks & Describe possible risks of harm to the participant. \\
\hline - Appropriate alternatives & Disclose other options that may be advantageous specific to procedures or possible treatments. \\
\hline - Confidentiality practices & State how records identifying the participant will be maintained. \\
\hline $\begin{array}{l}\text { Whether/how injury will be } \\
\text { compensated (only if study } \\
\text { exceeds minimal risk of harm) }\end{array}$ & Explain whether compensation is available to cover study related for medical treatment or other injury. \\
\hline - Study team contact & Identify who to contact if there are questions or to report a research related injury. \\
\hline - Voluntary nature of participation & $\begin{array}{l}\text { Make clear that participation is voluntary and that there is no penalty or loss of benefits if the individual chooses } \\
\text { not to participate or changes their mind after initial agreement to enroll. }\end{array}$ \\
\hline
\end{tabular}

The first step was to deconstruct the IRB-approved consent content by breaking it into segments that aligned with the federal requirements elements of informed consent to participate in research (see 46 CFR 46.116), (see Table 1). By doing this, we could focus on what communication was needed to comply with the federal regulations. Upon completion of this step, the research team discussed the challenges they faced while reading each segment and commented on the document length, technical language, and redundant information.

\section{Be People-Centered}

Unfortunately, in academic research, researchers are torn between making the consent accessible to those who may be recruited to participate in the research and adhering to the consent template that the IRB wants researchers to follow. The IRB-approved consent for selected for our use-case exceeded the recommended 6-8th grade reading level that IRB guidance suggests. As such, the next step involved our three researchers (MG, DK, EK) independently revised each segment of text with a goal of achieving a readability score of a 6-8th grade reading level. This participatory design process provides valuable insights as the researchers are engaged directly in the task of trying to develop consent language as a researcher would when applying our method to their consent communication process (19).

As noted, a norm of US human research ethics is to aim for a readability score that a majority of the adult population would be able to read, however, rarely is this goal achieved. A challenge was encountered by our team when attempting to revise language occurred when attempting to describe the technology intended for use in the research (passive sensor devices) along with the legal language that the IRB requires. Disclosure of reporting requirements is routine in some studies due to legal requirements like reporting mandates (e.g., disclosure of illegal behaviors like child or elder abuse). That was true for the consent serving as our use-case. The language required by the IRB to convey indemnification and mandated reporting was nearly impossible to reduce to an accessible reading level.
TABLE 2 | Readability analysis.

\begin{tabular}{lll}
\hline & $\begin{array}{l}\text { Original IRB approved } \\
\text { consent }\end{array}$ & $\begin{array}{l}\text { Rewritten consent by } \\
\text { research team }\end{array}$ \\
\hline Word count & 2,464 & 679 \\
Readability grade & $\begin{array}{l}\text { Microsoft Word: } 13.3 \\
\text { Online-Utility: } 11.91\end{array}$ & $\begin{array}{l}\text { Microsoft Word: } 9.3 \\
\text { Online-Utility: } 9.13\end{array}$
\end{tabular}

\section{Use an Activity-Centered Systems Approach}

Once each team member had revised the consent segment to the best of their ability, they reviewed all revisions to ensure alignment with the federal regulations and applied a readability software to assess grade level. The revised segments were analyzed using a readability feature in Microsoft Word, since that tool was compatible for analyzing smaller text segments and accessible to the team. The team members then compared the different versions of text and chose the version that achieved the lowest grade level.

Further iterations were needed to reach the 6-8th grade reading level. The team then consulted with an external readability expert well-versed in revising informed consent documents into "plain language" (20). The external consultant used Readability Studio 1.1 to assess the IRB-approved consent form, which provides grade and difficulty level along with suggestions for how to further simplify the language. The team implemented the suggested wording and finalized the revised document. The final step involved inserting visual images of the technology and graphics to complement the text and improve readability.

\section{Rapid Iterations of Prototyping and Testing}

The last step involved applying the Digital Health Checklist (DHC) consent prototype tool to identify areas where the consent content could be expanded to address the four domains of Access and Usability, Privacy, Risks and Benefits, and Data Management. Each of the four domains are expanded in the "respect for persons" row of the checklist matrix, which corresponds to what a researcher should consider when developing the informed 
consent document so that specific information about a digital health strategy/tool can be addressed. Not all of the checklist prompts will be relevant but, the checklist facilitates reflection of what might have been overlooked-particularly if relying on an IRB consent template to guide content. Our team compared what was in the IRB-approved and subsequently revised consent form to the DHC and identified content areas that would need to be added to improve the consent for use in this digital health study.

\section{RESULTS}

\section{Readability and Content}

The original IRB-approved consent form and the revised text were analyzed internally using both Microsoft Word and OnlineUtility 1.1, which provide average readability and grade level scores between the two software (see Table 2). The results of the IRB-approved consent showed a 12.6 reading level, whereas the revised language by our study team received $12.4,12$, and 12.58 , respectively. The final consent document synthesized the most readable of the three revised versions. We then incorporated language recommended by the software tool for improving readability, which resulted in a final revised consent readability score of a 9.2 grade level. Table 2 . Readability analyses and word count of original IRB-approved consent form and final revised version.

For a more detailed example of how the text was modified to improve readability, see Figures 2, 3 below which illustrate examples of a paragraph in the rewritten (Figure 2) vs. the original (Figure 3) consent form. This text focuses on risk management and how the study team will be trained to respect participant privacy. The original text was 100 words, and the sentences were much longer when compared to the revised version by 52 words.

The research team was able to improve the readability and lower the reading level of the passages by 3 grade-levels from the original version, however, did not achieve the targeted 68 th grade reading level. This was achieved by following the plain language guidelines published by the US government ${ }^{2}$, which includes using words with fewer syllables, shorter sentences and shorter paragraphs.

\section{Presentation and Visuals}

The final revised version of the consent was augmented to include graphics and pictures of the digital tool. See Figures 4, 5 to compare presentation and visuals of the revised (Figure 4) and original (Figure 5) versions of the consent forms.

The DHC tool was then referenced to identify consent content that could be expanded to address areas specific to Access and Usability, Privacy, Risks and Benefits, and Data Management in the revised consent form. Table 3 illustrates the four domains and their prompts that were used to evaluate the consent form. Under the Access and Usability domain, all the statements noted in the DHC tool were addressed in both the original and revised consent document. Under the Risks and Benefits domain, which covers potential harms and impact with respect to possible benefits, 10 of the 12 statements were addressed. Under the Privacy domain, which covers the extent, purpose, and sharing of personal data, two of the seven statements were addressed in the original consent and carried forward to the revised consent. Under the Data Management domain, two of the 10 checklist prompts were addressed.

While the original consent included basic information specific to Access and Usability deemed necessary for informed consent to occur, the other three domains were lacking. The next iteration of the consent form will be revised to include information about reputational and unknown harms as well as specify why personal data are being collected and where data are stored and the extent of 3rd party access. Moreover, the possibility of a bystander being recorded is important to address and was not included in the original consent. Bystanders are not typically considered when consenting a research participant but, is increasingly important given the passive and pervasive nature of sensor technologies. Given the consent used in this exercise described a study that used a wearable microphone, addressing bystander

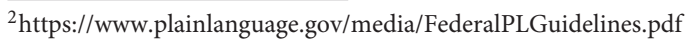

The study team will be trained to stop listening and delete portions of the recordings with private info such as

finances, substance use, and medical diagnosis. Risks are no more than what you and your child will

experience daily. Minor difficulties include stopping your child from removing the recorder from their clothes.

FIGURE 2 | Example of a paragraph in the rewritten version consent form.

\begin{abstract}
During the home device recordings, you are free to pause or stop the device at any time. Upon review of the audio files, our research staff will be trained to immediately stop listening to and delete any portions of an audio recording revealing private information (e.g., discussion of financial matters, drug/alcohol use, medical diagnoses). Your child is at potential risk for any typical injury (e.g., while at play) if your child were to fall on the recorder. You may also experience a slight inconvenience in your daily routine to ensure your child does not remove the recorder from his/her clothing.
\end{abstract}

FIGURE 3 | Example of a paragraph in the original IRB-approved consent form. 


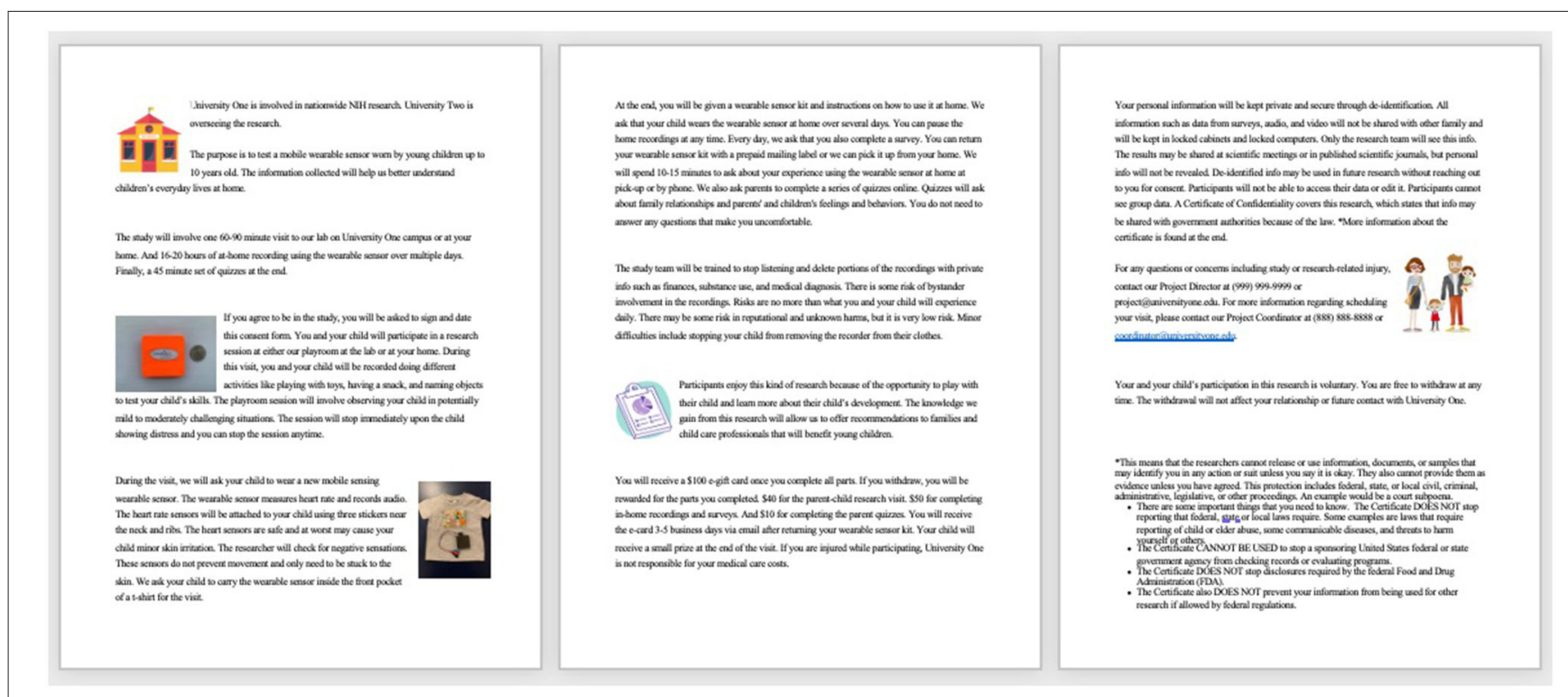

FIGURE 4 | Rewritten version of consent form by research team that includes all required elements of informed consent.

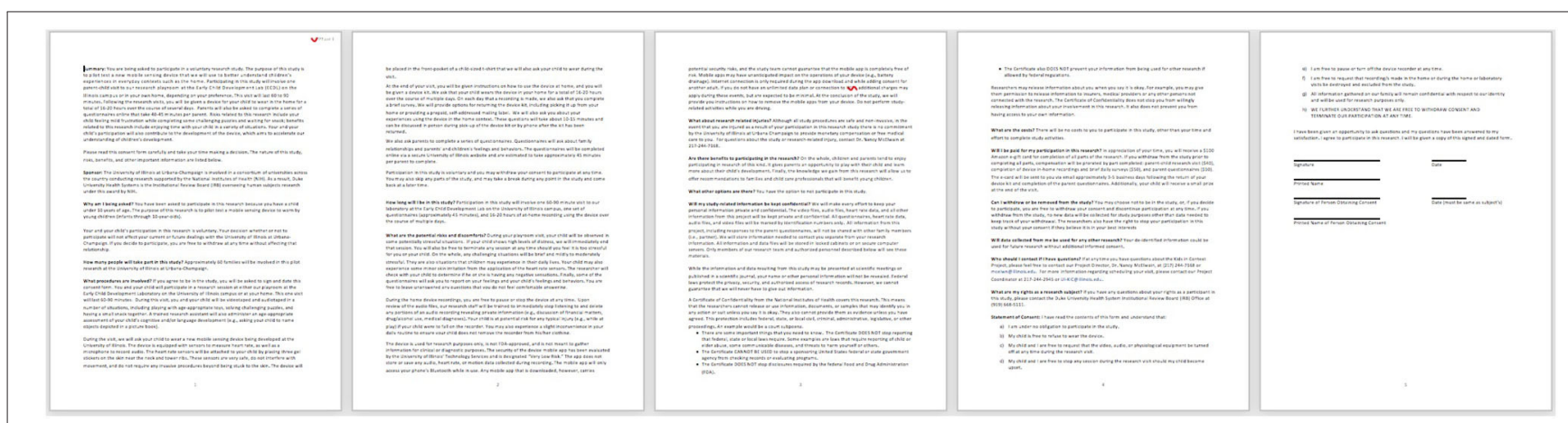

FIGURE 5 | Original IRB approved consent form approved by an IRB.

considerations is appropriate. Lastly, information about data practices including data transfer, storage, and sharing along with how much access participants will have to collected data will be included in the next iteration of the consent document. At that point, the consent form will include all recommendations in the DHC informed consent blueprint, be accessible in terms of reading grade level and will advance to the stage of further iterative design with prospective research participants.

\section{DISCUSSION}

The main objective of this paper was to provide a step-by-step description of developing an informed consent communication. Using a participatory design, we included researchers who are involved with creating consent communications but who have little experience. Few would argue that valid consent requires that a person be provided with adequate and relevant information.
Yet, the process of developing an informed consent document is typically guided by a template that the research ethics board provides for the purpose of helping a researcher create a document that complies with federal regulations and institutional practices. Unfortunately, the consent templates do not include guidance on how to make the consent language or presentation of information accessible or particularly meaningful.

What might make informed consent meaningful has been a subject of study though, whether it can be achieved in practice is uncertain. Dranseika et al. (21) suggested that researchers take the time to learn what information might be relevant for prospective participants and actually speak with patients to learn what might contribute to their decisions about participation in a study. Moreover, they called for empirical research to understand the concept of relevance and how consent content might vary depending on socio-economic and cultural background (21). Most empirical research on informed consent to date has focused on comprehension of the consent content and, subsequent 
understanding of the research. For example (22), designed and tested an instrument to assess participant objective and subjective understanding of a cancer clinical trial (22). Wilbanks (11) recognized that problems may exist in the traditional consent process and explored how the concept of a choice architecture (23) might be used in guiding decision to participate in digital research whereby consent information was presented on a personal mobile device (11). In fact, Wilbanks argued that in an era of technology mediated clinical and biomedical research with the associated volume, velocity and variety of data, that bioethics must meet the new demands. Experimenting with new design elements with a focus on linear, graphic/pictorial and brief narrative, the team at Sage Bionetworks created a consent flow that was used to communicate informed consent content via an iPhone. Similar to other studies, the need to engage people prospectively in the design process was a limitation. Formative research with mPower study participants conducted by (10) similar inconsistencies in understanding as would be found in traditional face to face consent but, highlighted a desire by participants to be partners in research (10).

The importance of engaging "end users," in this case researchers and, eventually research participants, early in the design phase of a consent design process cannot be understated. Applying human centered design principles to the concept of informed consent makes sense yet, there is limited literature on this topic. The exceptions are the work of (24) who published a conceptual model of design principles for informed consent related to cookie technology and web browser design (24) and Wilbank's work mentioned previously (11). Recognizing the need to move toward a meaningful and accessible informed consent communication for digital health research is what led to the design process described in this paper.

In this study, we have taken steps to bridge the gap in accessible and meaningful informed consent by moving beyond a transactional form to a presentation of information that is likely to be read and understood. An iterative process was used to create consent information that can be presented to a prospective research participant by first improving the document readability score and then aligning content with the Digital Health Checklist tool. By utilizing the DHC "respect for persons" consent prototype builder, we were able to guide alignment with the four domains of: Access and Useability, Privacy, Risks and Benefits and Data Management.

With this revised consent communication as a starting point, we now plan to engage prospective research participants in iterative consent design workshops to move toward the ideal of meaningful consent. The next phase of this research will involve people who may eventually participate in our larger HBCD study. They will be asked to comment on the relevance and clarity of the consent language. Building on the Digital Health Checklist and emerging work on participant-centered and dynamic consent models, we will include prompts for participants to rate the relevance of aspects of digital health research that are unique and challenging.

For this initial work, our goal is to help researcher understand and apply a process for conveying complex topics, via a consent communication using tools to make language accessible and content complete. Areas of interest expressed by researchers, which led, in part, to development of the DHC tool, are framed as "how might we" questions. Examples follow:

- Improve understanding of how the technology works?

- Convey individual and societal implications of the knowledge gained?

- Communicate how personal health information is transmitted and stored to the cloud?

- Describe differences between real-time data collection?

- Respect preferences for privacy and control of personal information?

- Understand the extent of control participants want with respect to managing data?

- Accurately convey how personalized algorithms work to nudge behavior change?

- Gauge acceptance of health technologies among family, coworkers and friends?

Clearly, informed consent to participate in digital health research has received little attention from a human centered design perspective. With increasing interest from large scale programs, like the All of Us Research Program and Patient Centered Outcomes Research Initiative, to engage with research participants as partners in the learning process, the opportunities are exciting. The ethical principle of "respect for persons" requires that we actually do more than create a transaction to demonstrate compliance between a researcher and participant. To authentically demonstrate "respect for persons" is to codesign the consent content and process to improve capacity among researchers so that the person considering study participation is informed and able to make a decision about whether to volunteer. Through a human centered design process, we can move from a transaction to a meaningful exchange of information that may lead to an informed consent in practice.

Our planned summative research will expand the work reported here. We encourage other researchers to replicate this process when creating their consent communications. While the results will vary since each study is unique in context, we are confident our methods, conveyed via an authentic use case, can serve as a concrete example.

\section{LIMITATIONS}

The informed consent prototype design process described here has not involved people external to our research team; however, we have confidence that our team is similar to those who would be eligible to enroll. Specifically, co-authors involved with the deconstruction exercise included two members of our research team (EK, DK) who had no prior experience writing or reviewing informed consent documents and one member (MG) who had limited experience with preparing research protocols for IRB review. The senior author $(\mathrm{CN})$ is a subject matter expert in research ethics and did not participate in the deconstruct/rebuild exercise. While we have taken the preliminary steps to make the IRB-approved consent more accessible via a lower readability score, we have not tested the language or obtained feedback on 
TABLE 3 | Digital health checklist consent blueprint: ethical principle "respect for persons" across four domains.

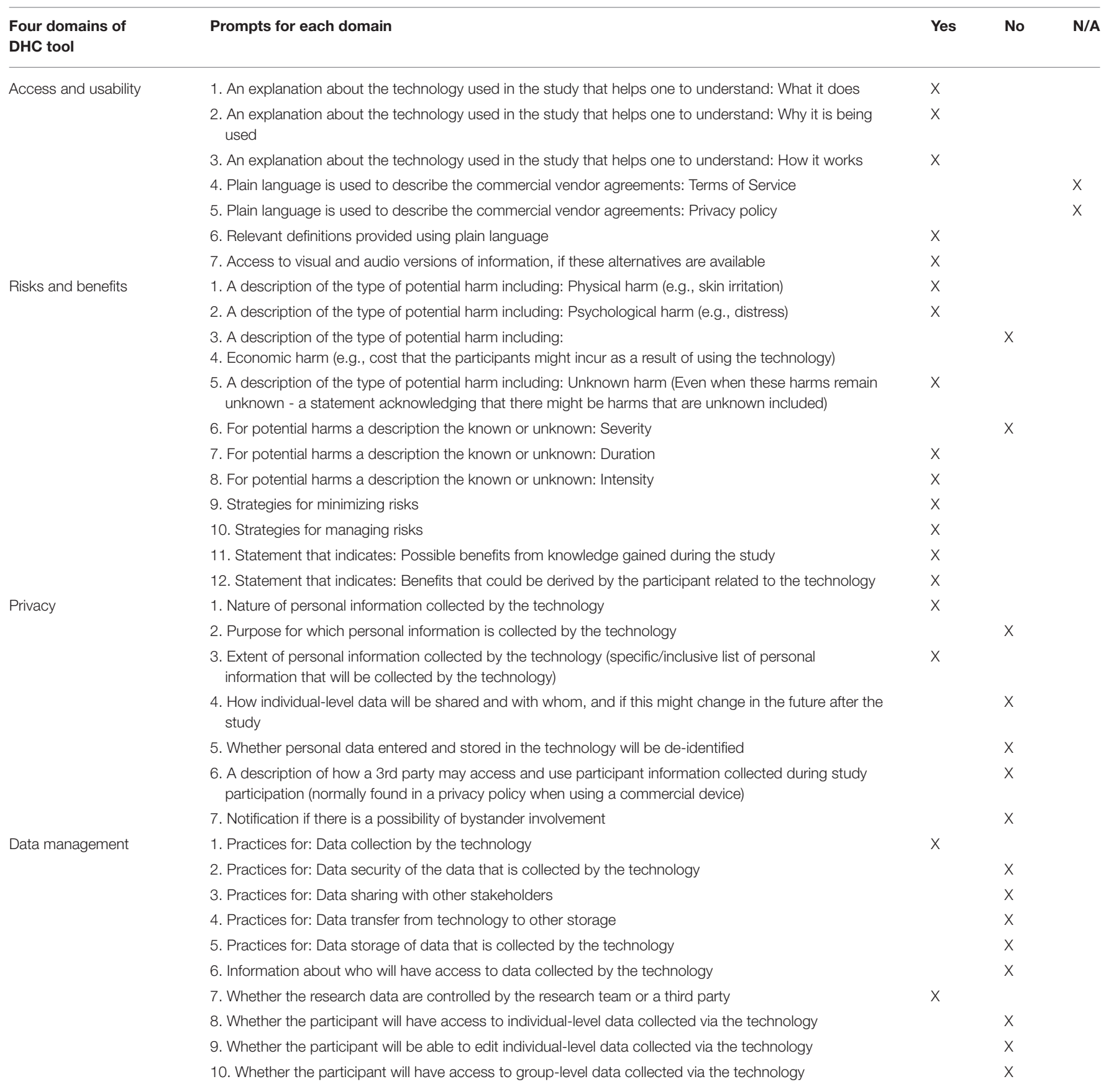

whether prospective participants find the additional information prompted by the Digital Health Checklist to be relevant or meaningful.

\section{CONCLUSION}

To achieve responsible digital health requires that we design our studies, to the extent possible, with our research participants and put their interests at the forefront. The wild west of the digital health era allows for exciting innovation and yet, without a purposeful philosophy of "respect for persons" at the core, we as a community of researchers, technologists, clinicians and citizens will make avoidable mistakes. This paper describes the initial steps that researchers can apply for creating an accessible informed consent for use in digital health research. By making information developed for prospective participants accessible, we can then take a human centered approach to learning what is relevant and how best to convey information that matters most to those we will include in future research studies. 


\section{DATA AVAILABILITY STATEMENT}

The original contributions presented in the study are included in the article/supplementary material, further inquiries can be directed to the corresponding authors.

\section{AUTHOR CONTRIBUTIONS}

$\mathrm{CN}$ conceptualized the project, led the design of prototype development and application of the Digital Health Checklist, and developed the first draft of the manuscript. DK and EK contributed to the consent analysis process, revisions using the readability, checklist tools and created figures, and tables used in this paper. MG led the co-authors (DK and EK) in deconstructing and revising the consent content and prototype development

\section{REFERENCES}

1. National Institutes of Health. The belmont report ethical principles and guidelines for the protection of human subjects of research. J Am Coll Dent. (1979) 81:4-13.

2. Office for Human Research, Protections. Federal Policy for the Protection of Human Subjects ('Common Rule). Regulations. (2016). Available online at: https://www.hhs.gov/ohrp/regulations-and-policy/regulations/index.html

3. Brody JL, Dalen J, Annett RD, Scherer DG, Turner CW. Conceptualizing the role of research literacy in advancing societal health. J Health Psychol. (2012) 17:724-30. doi: 10.1177/13591053114 25273

4. Appelbaum PS, Roth LH, Lidz C. The therapeutic misconception: informed consent in psychiatric research. Int J Law Psychiatry. (1982) 5:31929. doi: 10.1016/0160-2527(82)90026-7

5. Sugarman J, McCrory DC, Powell D, Krasny A. Empirical research on informed consent. An annotated bibliography. Has Cen Rep. (1999) 29:S1U1. doi: 10.2307/3528546

6. Palmer BW, Cassidy EL, Dunn LB, Spira AP, Sheikh JI. Effective use of consent forms and interactive questions in the consent process. IRB. (2008) 30: $8-12$.

7. Al-Sheyab NA, Alomari MA, Khabour OF, Shattnawi KK, Alzoubi $\mathrm{KH}$. Assent and consent in pediatric and adolescent research: school children\&rsquo;s perspectives. Adol Health Med Ther. (2019) 10:714. doi: 10.2147/AHMT.S185553

8. Bloss C, Nebeker C, Bietz M, Bae D, Bigby B, Devereaux M, et al. Reimagining human research protections for 21st century science. J Med Int Res. (2016) 18:e329. doi: 10.2196/jmir.6634

9. Bot BM, Suver C, Neto EC, Kellen M, Klein A, Bare C, et al. The MPower study, Parkinson disease mobile data collected using researchkit. Sci Data. (2016) 3:1-9. doi: 10.1038/sdata.2016.11

10. Doerr M, Truong AM, Bot BM, Wilbanks J, Suver C, Mangravite LM. Formative evaluation of participant experience with mobile econsent in the app-mediated parkinson mpower study: a mixed methods study. JMIR Mhealth Uhealth. (2017) 5:e14. doi: 10.2196/ mhealth.6521

11. Wilbanks J. Design issues in E-consent. J Law Med Ethics. (2018) 46:11018. doi: 10.1177/1073110518766025

12. Nebeker C, Bartlett Ellis RJ, Torous J. Development of a decision-making checklist tool to support technology selection in digital health research. Trans Behav Med. (2020) 10:1004-15. doi: 10.1093/tbm/ibz074

13. Dittrich D, Kenneally E. The menlo report: ethical principles guiding information communication technology research. U.S. Dep Home Secur. (2012) 1-17. doi: 10.2139/ssrn.2445102

14. Harrington N, Wei Z, Hernandez-Pacheco B, Biu Q, DeYoung PN, Duwaik B. et al. Bedscales: a non-contact adherence-independent multi-person and contributed to the methods and result sections of the manuscript. All authors contributed to the article and approved the submitted version.

\section{FUNDING}

This work was supported by National Institutes on Drug Abuse (NIDA) grant number: 3R34DA050341-01S3 bioethics supplement.

\section{ACKNOWLEDGMENTS}

We thank our colleague Dr. Mark Hochhauser, Readability Consultant, for his insights into writing a consent form at the 6-8th grade reading level. sensor for longitudinal physiologic monitoring in the home bed. BioRxiv. (2020). doi: 10.1101/2020.03.31.018523

15. Norman DA, Draper SW. User Centered System Design. Hillsdale, NJ: Lawrence Erlbaum Associates (1986).

16. Hadden KB, Prince LY, Moore TD, James LP, Holland JR, Trudeau CR. Improving readability of informed consents for research at an academic medical institution. J Clin Trans Sci. (2017) 1:361-5. doi: 10.1017/cts 2017.312

17. Samadi A, Asghari F. Readability of informed consent forms in clinical trials conducted in a skin research center. J Med Ethics Hist Med. (2016) 9:7.

18. Nebeker C, Harlow J, Espinoza Giacinto R, Orozco-Linares R, Bloss CS, Weibel N. Ethical and regulatory challenges of research using pervasive sensing and other emerging technologies: IRB perspectives. AJOB Emp Bio. (2017) 8:266-76. doi: 10.1080/23294515.2017.1403980

19. Jorgensen DL. Participant observation. In: Scott RA, Kosslyn SM, editors. Emerging Trends in the Social and Behavioral Sciences. (2015). doi: 10.1002/9781118900772.etrds0247

20. Stableford S, Mettger W. Plain language: a strategic response to the health literacy challenge. J Public Health Policy. (2007) 28:71-93. doi: 10.1057/palgrave.jphp.3200102

21. Dranseika V, Piasecki J, Waligora M. Relevant information and informed consent in research: in defense of the subjective standard of disclosure. Sci Eng Ethics. (2017) 23:215-25. doi: 10.1007/s11948-0169755-4

22. Joffe S, Cook EF, Cleary PD, Clark JW, Weeks JC. Quality of informed consent: a new measure of understanding among research subjects. JNCI J Natio Can Inst. (2001) 93:139-47. doi: 10.1093/jnci/93.2.139

23. Thaler RH, Sunstein CR, Balz JP. Choice architecture. SSRN Electr J. (2010) 428-39. doi: 10.2139/ssrn.1583509

24. Millett LI, Friedman B, Felten E. Cookies and web browser design: toward realizing informed consent online. In: Proceedings of the SIGCHI Conference on Human Factors in Computing Systems (2001). p. 46-52.

Conflict of Interest: The authors declare that the research was conducted in the absence of any commercial or financial relationships that could be construed as a potential conflict of interest.

Copyright (C) 2021 Nebeker, Gholami, Kareem and Kim. This is an open-access article distributed under the terms of the Creative Commons Attribution License (CC BY). The use, distribution or reproduction in other forums is permitted, provided the original author(s) and the copyright owner(s) are credited and that the original publication in this journal is cited, in accordance with accepted academic practice. No use, distribution or reproduction is permitted which does not comply with these terms. 